\title{
Natural history of adult-onset Ménétrier's disease: Report of a case with 9-year follow-up
}

\author{
LI-SHOU XIONG ${ }^{1}$ and YING-YING GONG ${ }^{2}$ \\ Departments of ${ }^{1}$ Gastroenterology and ${ }^{2}$ Geriatrics, The First Affiliated Hospital of Sun Yat-sen University, \\ Guangzhou, Guangdong 510080, P.R. China
}

Received January 20, 2015; Accepted February 25, 2016

DOI: $10.3892 /$ etm.2016.3181

\begin{abstract}
Ménétrier's disease (MD) is a rare disease characterized by markedly hypertrophied gastric mucosal folds typically associated with hypoalbuminemia and anemia. However, the natural history of MD in adults remains unclear and is rarely reported in the literature. The current study presents a case of MD with a 9-year follow-up. A 56-year-old man was diagnosed with MD in 2005. The patient was followed up and underwent surveillance endoscopy once or twice each year. In the present case, the anemia and hypoproteinemia were eliminated following red blood cell transfusion and intravenous iron therapies. The symptoms were relieved after 4 years. Treatment with octreotide had little effect on the gastric mucosa, while antimicrobial combination therapy provided no benefit in the present $H$. pylori-negative case of MD. In addition, despite abnormalities of the gastric mucosa in the patient persisting after 9 years of follow-up with no evidence of malignancy, malignant transformation in MD should not be overlooked, and regular monitoring of the gastric mucosa via endoscopy is necessary.
\end{abstract}

\section{Introduction}

Ménétrier's disease (MD), also known as hypertrophic protein-losing gastropathy, was first described in 1888 by Pierre Ménétrier (1). It is a rare clinical entity of unknown etiology characterized by large gastric folds associated with epithelial hyperplasia. As MD is a rare disease, incidence and mortality rate data remain undetermined. Primarily observed in male adults (mean age at diagnosis is 55 years), MD presents with variable symptoms. Classic presentation includes abdominal pain, nausea, vomiting, anemia, hypochlorhydria

Correspondence to: Professor Li-Shou Xiong, Department of Gastroenterology, The First Affiliated Hospital of Sun Yat-sen University, 58 Zhongshan II Road, Guangzhou, Guangdong 510080, P.R. China

E-mail: xionglishou@263.net

Key words: Ménétrier's disease, natural history, treatment, follow-up and peripheral oedema, which is due to protein loss across the gastric mucosa (2). The natural history of the disease may vary considerably between adults and children. In childhood, the disorder is typically arises suddenly and resolves spontaneously, and has been associated with cytomegalovirus (CMV) infection (2). By contrast, in adults, the course of the disease is progressive with an insidious onset (3). The aetiology of MD remains unknown, although previous studies have demonstrated that MD is associated with infections, including Helicobacter pylori, CMV and herpes simplex virus $(2,4,5)$. Furthermore, it has been shown that the pathogenesis of MD is associated with the upregulation of transforming growth factor (TGF)- $\alpha$ binding to gastric epidermal growth factor receptor (EGF-R) (6). However, targeted therapies such as $H$. pylori eradication and treatment with antibiotics, prednisone, octreotide and monoclonal antibodies have yielded inconsistent results in clinical trials (7-9). Notably, a number of patients with MD have presented gastric cancer, which may demonstrate that MD carries an increased risk of cancer $(3,10)$. However, whether MD is a precancerous condition is yet to be elucidated. Due to the low incidence of MD, a limited amount of research has been performed to address the disease progression, its tendency to be refractory and its association with gastric cancer. In the current study, a case of adult-onset MD with a 9-year follow-up is presented.

\section{Case report}

In August 2005, a 56-year-old man was admitted to The First Affiliated Hospital of Sun Yat-sen University (Guangzhou, China) with recurrent upper abdominal pain, bloating, acid regurgitation, heartburn, hematochezia and weight loss of $7 \mathrm{~kg}$ experienced for a duration of 1 year. The patient's initial symptom of upper abdominal pain had occurred from April 2004 and the patient had undergone an endoscopy on April 28, 2004, which only revealed chronic non-atrophic gastritis. Subsequently, the patient used Chinese herbal medicine to control the symptoms; however, the categories of and ingredients within the traditional Chinese medicines are unknown. The patient's medical history included hyperlipemia and benign prostatic hyperplasia. The patient had been a smoker for 20 years prior to quitting smoking in 2004, and denied drinking alcohol. The family history was considered to be noncontributory. 
Upon physical examination the patient's face was pale and no pretibial edema was detected. Laboratory studies indicated iron-deficiency anemia (hemoglobin, $59 \mathrm{~g} / 1$; normal range, $120-160 \mathrm{~g} / \mathrm{l}$ ) and hypoalbuminemia (albumin, $31 \mathrm{~g} / \mathrm{l}$; normal range, 35-50 g/l), as displayed in Table I. Serum tumor markers including carcinoembryonic antigen, $\alpha$-fetoprotein, carbohydrate antigen (CA)125 and CA199 were within normal ranges. To exclude Zollinger-Ellison syndrome, serum gastrin levels were assessed and revealed to be normal. A chest X-ray and abdominal ultrasound were normal, as were the results of an electrocardiogram. Upper gastrointestinal endoscopy was subsequently performed with an Olympus GIF-Q180 gastroscope (Olympus Corporation, Tokyo, Japan), which revealed large edematous gastric folds throughout the fundus and body, with areas of superficial punctate erosions and exudate, but sparing the antrum and pylorus (Fig. 1A). The stomach was poorly distensible. Endoscopic ultrasound of the stomach was also performed (Olympus GF-UE260-AL5) and the results are displayed in Fig. 2A.

To obtain more accurate histopathological evidence, an endoscopic mucosal resection (EMR) was performed in the body of the stomach. Briefly, the slices were fixed in 10\% neutral buffered formalin (Sigma-Aldrich, St. Louis, MO, USA) for $\geq 24 \mathrm{~h}$ and subsequently embedded in paraffin wax (Wuhan Jiayuan Quantum Dots Co., Ltd., Wuhan, China). Following dehydration with 95-75\% absolute ethyl alcohol (Wuhan Jiayuan Quantum Dots Co., Ltd.), the slices were stained with hematoxylin (Sigma-Aldrich) for $10 \mathrm{~min}$, soaked in water for $5 \mathrm{~min}$, rested in $1 \%$ hydrochloric acid alcohol (Wuhan Jiayuan Quantum Dots Co., Ltd.) for $3 \mathrm{sec}$ and rinsed with water for 20 min prior to staining with eosin solution (Sigma-Aldrich) for $10 \mathrm{~min}$. Subsequently, the slices were dehydrated with a graded alcohol series and sealed with half a drop of neutral balsam (Wuhan Jiayuan Quantum Dots Co., Ltd.). Stained slices were observed under an Olympus BX-43 optical microscope (magnification, x400; Olympus Corporation, Tokyo, Japan). Each slice was randomly selected from five non-overlapping fields of view, and double blind observation was conducted by two pathologists, respectively. H. pylori was undetectable. Therefore, a diagnosis of MD was concluded based upon the sizeable expansion of the mucosa and foveolar hyperplasia with a reduced number of parietal cells and chief cells (Fig. 2B).

The patient received a transfusion of red blood cell concentrate $(600 \mathrm{ml})$ and was administered oral folic acid (10 $\mathrm{mg}$ three times a day) and polysaccharide-iron complex capsules ( $0.15 \mathrm{~g}$ daily) to treat his anemia. In order to control the patient's symptoms of recurrent upper abdominal pain, bloating, acid regurgitation, heartburn and hematochezia, esomeprazole (20 mg daily) was administered. Three weeks later, the patient's hypoalbuminemia was resolved after an albumin level of $37 \mathrm{~g} / 1$ was reached, and hemoglobin levels increased to $87 \mathrm{~g} / 1$. The patient was discharged as an outpatient and received follow-up by surveillance endoscopy once or twice per year. From August 2005 to June 2007, the patient displayed hemoglobin levels ranging from 60 to $85 \mathrm{~g} / 1$ and received no blood transfusions. The symptom of upper abdominal pain recurred occasionally. Using an ISOMAX 2000 Carbon $^{13}$ breath infrared spectrometer (Isodiagnostika, Inc., Edmonton, $\mathrm{AB}, \mathrm{Canada}), \mathrm{C}^{13}$ urea breath test was performed

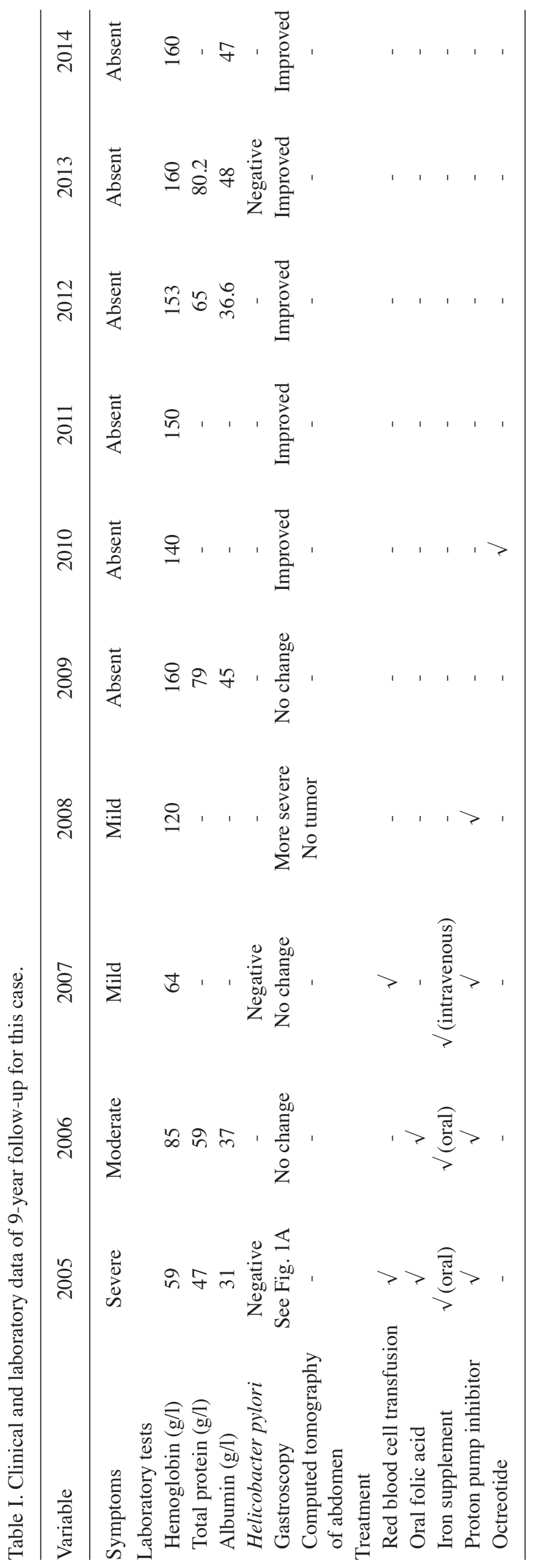



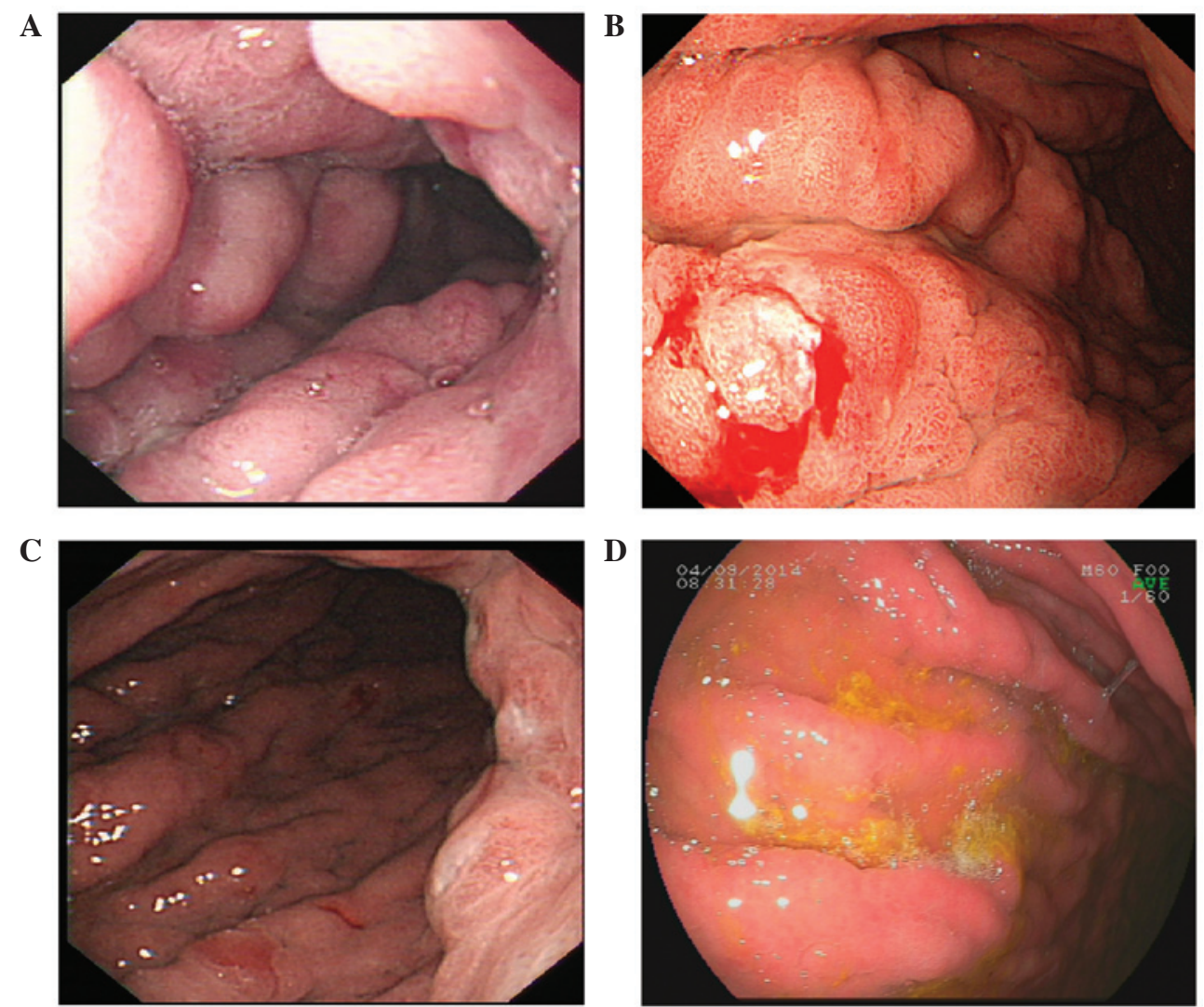

Figure 1. Endoscopic manifestations. (A) Endoscopy in 2005 revealed large gastric rugal folds in the body of the stomach with areas of superficial punctate erosions and erythema. (B) Endoscopy in 2008 indicated larger gastric folds of the stomach body with nodularity, erosions and hemorrhage. (C) Endoscopy in 2009 showed that the giant gastric folds in the body of the stomach had not improved. (D) Final surveillance endoscopy in 2014 displayed slight improvements in the gastric folds of the stomach body, but a persistence of the abnormalities of the gastric mucosa.
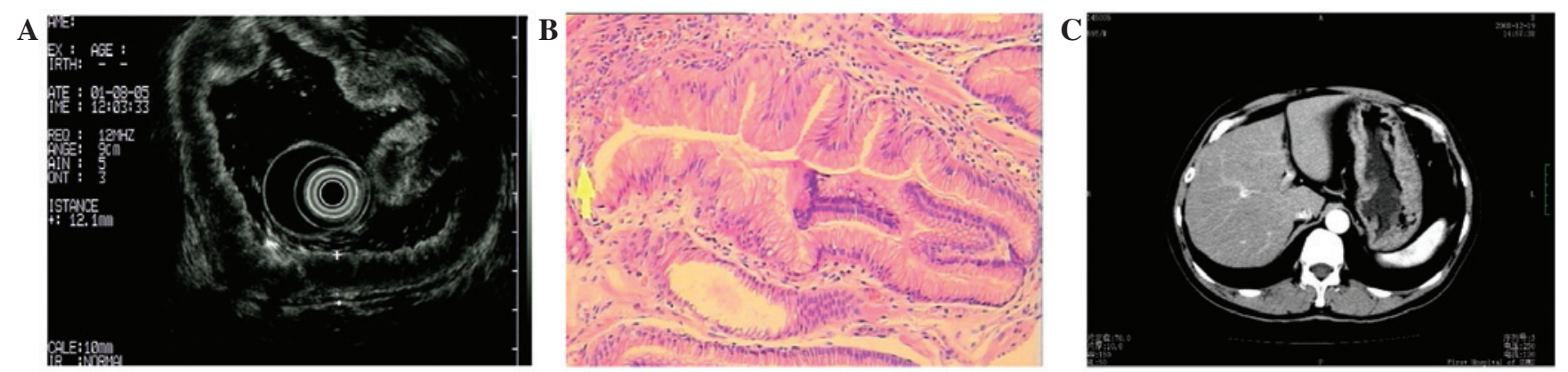

Figure 2. Imaging and histopathology. (A) Endoscopic ultrasound of the stomach demonstrated diffuse and regular gastric wall thickening of the second layer corresponding to the mucosa, with preservation of wall stratification. The thickest wall of the stomach was $12.1 \mathrm{~mm}$. (B) Histopathological analysis of endoscopic mucosal resection biopsy with hematoxylin and eosin staining showed thickening of the mucosa and foveolar hyperplasia. Atrophy of the parietal cells and chief cells was observed. The lamina propria exhibited only mild chronic inflammation. Magnification, x400. (C) Contrast-enhanced computerized tomography of the abdomen in 2008 revealed significantly thickened gastric mucosa and fold formation of the gastric body with no mass and no mesenteric lymph node swelling, which indicated no signs of gastric carcinoma.

for a second time to detect $H$. pylori during this period and returned negative. It was hypothesized that this result may have been influenced by the use of esomeprazole. The patient was treated empirically for $H$. pylori with a 14-day course of amoxicillin ( $1 \mathrm{~g}$ twice a day), clarithromycin $(0.5 \mathrm{~g}$ twice a day) and esomeprazole (20 $\mathrm{mg}$ twice a day).

On November 7, 2007, the patient was admitted again as a result of anemia with hemoglobin levels of $64 \mathrm{~g} / \mathrm{l}$. Serum concentrations of folic acid and vitamin $\mathrm{B}_{12}$ were within the normal range with the exception of ferritin $(1.00 \mu \mathrm{g} / \mathrm{l}$; normal range, 16.4-323 $\mu \mathrm{g} / \mathrm{l})$. Subsequently, the patient received another red blood cell concentrate transfusion $(400 \mathrm{ml})$, the administration of oral folic acid was discontinued and iron dextran was injected intravenously for 1 week ( $100 \mathrm{mg} /$ day). After 3 weeks, hemoglobin levels recovered to $126 \mathrm{~g} / 1$. An endoscopy was performed again on November 8,2007, revealing no changes in the large gastric folds and no evidence of gastric malignancy.

Subsequently, the patient displayed hemoglobin levels $>120 \mathrm{~g} / \mathrm{l}$ and felt well. An endoscopy on December 17, 2008, revealed that the large edematous gastric folds of the stomach 
body were more severe than they had previously been (Fig. 1B); however, the gastric biopsies revealed no carcinoma. There was a concern that the random biopsies may not have decisively ruled out the possibility of a carcinoma. Thus, to rule out gastric carcinoma with greater certainty, contrast-enhanced computerized tomography of the abdomen was performed on December 19, 2008, and showed significant thickening of the gastric body and fundus with no mass and no mesenteric lymph nodes swelling (Fig. 2C). It was found that the administration of $20 \mathrm{mg}$ esomeprazole as required was sufficient to control the symptoms. The patient had gained $6 \mathrm{~kg}$ in weight and reported no symptoms on July 29, 2009; however, the endoscopy revealed no signs of improvement (Fig. 1C). The oral administration of esomeprazole was discontinued at this stage, based on the lack of symptoms and weight gain.

On the basis of preliminary reports detailing the use of octreotide for the successful treatment of Ménétrier's disease (11), three doses of octreotide acetate injection (20 mg/month) were administered the patient in the present study from January to March 2010, which were tolerated well. However, a repeat endoscopy on October 13, 2010, revealed no remission of the gastric mucosa. The final follow-up was performed on September 4, 2014. The findings on endoscopy indicated a slight improvement in the gastric mucosa compared with previous results (Fig. 1D). All other clinical and laboratory data during 9-year follow-up are summarized in Table I.

\section{Discussion}

MD is a disease which predominantly presents in adulthood with markedly hypertrophied gastric mucosal folds, and is typically associated with hypoalbuminemia and anemia (12). In the current study, a case of MD is presented with a 9-year follow-up period. As the majority of cases of MD in pediatric patients are typically benign and self-limited with symptoms resolving within 5 weeks (13), the treatment is predominately supportive. However, the disease in adults is associated with considerable morbidity and even mortality, associated with surgical resection and a potential risk of malignant transformation $(3,10)$. Targeted therapies such as $H$. pylori eradication, antibiotics, prednisone, octreotide and monoclonal antibodies have yielded differing benefits in previous studies (7-9). There remains a lack of research to address the disease progression, refractory tendency and association with gastric cancer following treatment. Thus, a follow-up subsequent to treatment for MD is required.

Patients with MD may be debilitated as a result of epigastric pain, vomiting, weight loss, hypoalbuminemia and edema (12). In addition, previous cases of MD have also presented with severe iron-deficiency anemia $(14,15)$. In the present case, anemia and hypoproteinemia were successfully eliminated by red cell transfusion and intravenous iron therapies. On the basis of the rapid response, it is hypothesized that there may be possible iron and protein leakage from large folds and disruption of the iron absorption function due to inflammatory changes in the mucosa of stomach. The iron deficiency may alter mucosal function, which leads to protein leakage. Therefore, intravenous iron therapy rather than oral iron therapy is recommended in cases of MD with iron-deficiency anemia.
The etiology of MD remains as of yet largely unknown. One prominent report indicated that $>90 \%$ of cases displayed H. pylori following biopsies in 138 patients with hypertrophic gastropathy (16). Following the initial successful treatment of MD with cephalexin, several studies have reported that the eradication of $H$. pylori may lead to complete clinical and morphologic recovery $(15,17,18)$. Thus, mounting evidence suggests that $H$. pylori serves an etiological role in MD. However, H. pylori was not detected in the present case and further empirical treatment for $H$. pylori did not affect endoscopic morphology. It is indicated that $H$. pylori may not be a precursor factor in the disease process, but rather a contributing factor involved in the progression of the disease. Furthermore, in cases of $H$. pylori-negative MD, the refractoriness of the disease is to be expected following antimicrobial combination therapy. Notably, the patient in the present study had taken Chinese herbal medicine for 1 year prior to diagnosis. The results of the endoscopy the patient underwent prior to taking Chinese herbal medicine revealed no morphologic indications of MD. Whether Chinese herbal medicine had a casual role in the pathogenesis of the present case was unclear. However, it is possible that an infection or other as yet unknown cause may trigger an immunologic reaction through the action of cytokines such as TGF- $\alpha$. The continuous antigenic stimuli may lead to the development of MD.

Overproduction of TGF- $\alpha$, one of several ligands of the EGF-R, in the stomach has been suggested to explain several clinical features of MD, including decreased acid production, increased hyperplasia of surface mucous cells, oxyntic atrophy and increased mucin production (6). Molecular evidence suggests that octreotide may modulate the EGF-R signaling pathway $(19,20)$. On the basis of this molecular mechanism, and the successful use of octreotide in adults with MD $(7,11)$, the use of octreotide in the present study was prompted. However, the repeat endoscopy indicated that the large gastric folds were only marginally improved compared with the earlier endoscopy, but no remission of the abnormalities of the gastric mucosa. In 2000, Burdick et al (8) reported a severe case of MD that was unresponsive to numerous therapies, including octreotide, but was successfully treated with a monoclonal antibody against EGF-R, cetuximab. Since then, a number of clinical trials have reported the successful use of cetuximab in MD. Thus, in the present case, cetuximab therapy may be considered as a subsequent treatment.

Follow-up for patients with MD is essential as there is an increased risk of gastric-associated malignancies, primarily gastric carcinoma and gastric lymphoma $(3,10)$. This provides evidence for the hypothesis that MD is a premalignant lesion. However, the association with cancer has yet to be confirmed due to the low incidence rate of MD. Despite this limitation, it is recommended that patients are treated with particular attention and that lesions are monitored with regular endoscopic biopsy examination. In the present case, the patient received a surveillance endoscopy 1-2 times per year. There was no indication of gastric carcinoma in the following 9 years. Typically, in the cases that have been presented in the literature, MD and carcinoma were diagnosed simultaneously $(21,22)$. However, Wood et al (23) reported a patient with early gastric cancer 3.5 years subsequent to a diagnosis of $\mathrm{MD}$ and another report described a case that presented 
after 13 years (24). The longest follow-up period for a case of MD was 16 years, until the patient succumbed to mortality as a result of hepatocellular carcinoma (3). Therefore, although malignant transformation of this lesion remains controversial, patients should be followed up for a substantial period of time. If the patient is concerned about the potential development of cancer, a partial or total gastrectomy is recommended.

MD is a rare type of hypertrophic gastropathy and currently does not have a gold-standard therapy. In the present case, it is suggested that intravenous iron therapy rather than oral iron therapy is preferable in MD with iron-deficiency anemia. Treatment with octreotide had little effect on the gastric mucosa, while antimicrobial combination therapy provided no benefit in $H$. pylori-negative MD. Furthermore, although there was no evidence of carcinoma in the present patient at 9-year follow-up, malignant transformations in MD should not be overlooked, and it is necessary to monitor the gastric mucosa via routine endoscopy.

\section{References}

1. Coffey RJ Jr and Tanksley J: Pierre Ménétrier's and his disease. Trans Am Clin Climatol Assoc 123: 126-134, 2012.

2. Eisenstat DD, Griffiths AM, Cutz E, Petric M and Drumm B: Acute cytomegalovirus infection in a child with Ménétrier's disease. Gastroenterology 109: 592-595, 1995.

3. Scharschmidt BF: The natural history of hypertrophic gastrophy (Menetrier's disease). Report of a case with 16 year follow-up and review of 120 cases from the literature. Am J Med 63: 644-652, 1977.

4. Badov D, Lambert JR, Finlay $M$ and Balazs ND: Helicobacter pylori as a pathogenic factor in Menetrier's disease. Am J Gastroenterol 93: 1976-1979, 1998.

5. Jun DW, Kim DH, Kim SH, Song MH, Lee HH, Kim SH, Jo YJ and Park YS: Menetrier's disease associated with herpes infection: Response to treatment with acyclovir. Gastrointest Endosc 65: 1092-1095, 2007.

6. Dempsey PJ, Goldenring JR, Soroka CJ, Modlin IM, McClure RW, Lind CD, Ahlquist DA, Pittelkow MR, Lee DC, Sandgren EP, et al: Possible role of transforming growth factor alpha in the pathogenesis of Ménétrier's disease: Supportive evidence form humans and transgenic mice. Gastroenterology 103: 1950-1963, 1992.

7. Yeaton P and Frierson HF Jr: Octreotide reduces enteral protein losses in Ménétrier's disease. Am J Gastroenterol 88 95-98, 1993.

8. Burdick JS, Chung E, Tanner G, Sun M, Paciga JE, Cheng JQ, Washington K, Goldenring JR and Coffey RJ: Treatment of Ménétrier's disease with a monoclonal antibody against the epidermal growth factor receptor. N Engl J Med 343: 1697-1701, 2000.

9. Di Vita G, Patti R, Aragona F, Leo P and Montalto G: Resolution of Ménétrier's disease after Helicobacter pylori eradicating therapy. Dig Dis 19: 179-183, 2001.
10. Mosnier JF, Flejou JF, Amouyal G, Gayet B, Molas G, Henin D and Potet F: Hypertrophic gastropathy with gastric adenocarcinoma: Menetrier's disease and lymphocytic gastritis? Gut 32: 1565-1567, 1991

11. Rothenberg M, Pai R and Stuart K: Successful use of octreotide to treat Ménétrier's disease: A rare cause of abdominal pain, weight loss, edema, and hypoalbuminemia. Dig Dis Sci 54: 1403-1407, 2009

12. Rich A, Toro TZ, Tanksley J, Fiske WH, Lind CD, Ayers GD, Piessevaux H, Washington MK and Coffey RJ: Distinguishing Ménétrier's disease from its mimics. Gut 59: 1617-1624, 2010.

13. Kaneko T, Akamatsu T, Gotoh A, Shimodaira K, Shimizu T, Kiyosawa K, Katsuyama $\mathrm{T}$ and Momose A: Remission of Menetrier's disease after a prolonged period with therapeutic eradication of Helicobacter pylori. Am J Gastroenterol 94: 272-273, 1999.

14. Singh AK, Cumaraswamy RC and Corrin B: Diffuse hypertrophy of gastric mucosa (Menetrier's disease) and iron-deficiency anaemia. Gut 10: 735-737, 1969.

15. Yoshimura M, Hirai M, Tanaka N, Kasahara $Y$ and Hosokawa O: Remission of severe anemia persisting for over 20 years after eradication of Helicobacter pylori in cases of Menetrier's disease and atrophic gastritis: Helicobacter pylori as a pathogenic factor in iron-deficiency anemia. Intern Med 42: 971-977, 2003.

16. Bayerdorffer E, Ritter MM, Hatz R, Brooks W and Stolte M: Menetrier's disease and Helicobacter pylori. N Engl J Med 329: 60, 1993.

17. Kawasaki M, Hizawa K, Aoyagi K, Nakamura S and Fujishima M: Ménétrier's disease associated with Helicobacter pylori infection: Resolution of enlarged gastric folds and hypoproteinemia after antibacterial treatment. Am J Gastroenterol 92: 1909-1912, 1997.

18. Raderer M, Oberhuber G, Templ E, Wagner L, Pötzi R, Wrba F, Hejna $M$ and Base W: Successful symptomatic management of a patient with Ménétrier's disease with long-term antibiotic treatment. Digestion 60: 358-362, 1999.

19. Pinski J, Halmos G and Schally AV: Somatostatin analog RC-160 and bombesin/gastrin-releasing peptide antagonist RC-3095 inhibit the growth of androgen-independent DU-145 human prostate cancer line in nude mice. Cancer Lett 71: 189-196, 1993.

20. Watt HL, Kharmate GD and Kumar U: Somatostatin receptors 1 and 5 heterodimerize with epidermal growth factor receptor: Agonist-dependent modulation of the downstream MAPK signalling pathway in breast cancer cells. Cell Signal 21: 428-439, 2009.

21. Vandenborre KM, Ghillebert GL, Rutgeerts LJ, Geboes KR, Rutgeerts PJ, Verbanck JJ and Tanghe WR: Hypertrophic lymphocytic gastritis with a gastric carcinoma. Eur J Gastroenterol Hepatol 10: 797-801, 1998.

22. Choi SB, Park SS, Oh SY, Kim JH, Kim WB, Lee JH, Choi JW, Kim SJ, Kim CS and Mok YJ: Primary squamous cell carcinoma of the stomach that developed with Menetrier's disease. Dig Dis Sci 52: 1722-1724, 2007.

23. Wood MG, Bates C, Brown RC and Losowsky MS: Intramucosal carcinoma of the gastric antrum complicating Menetrier's disease. J Clin Pathol 36: 1071-1075, 1983.

24. Ramia JM, Sancho E, Lozano O, Santos JM and Domínguez F: Ménétrier's disease and gastric cancer. Cir Esp 81: 153-154, 2007 (In Spanish). 